\title{
Congenital heart disease in spondylothoracic dysostosis: two familial cases
}

\author{
J M Simpson, A Cook, N L K Fagg, N A MacLachlan, G K Sharland
}

\begin{abstract}
Two familial cases of spondylothoracic dysostosis are reported. Both cases had severe congenital heart disease in addition to the skeletal malformations which are characteristic of the condition.
\end{abstract}

(f Med Genet 1995;32:633-635)

Spondylothoracic dysostosis (Jarcho-Levin syndrome) is an autosomal recessive skeletal dysplasia characterised by severe abnormalities of the spine and ribs. Other features of the syndrome include short trunk, short neck, urogenital abnormalities, and anal abnormalities. The differentiation of spondylothoracic dysostosis from closely related disorders such as spondylocostal dysostosis is not entirely clear: classification of subtypes has been reviewed elsewhere. ${ }^{1}$ The prognosis of these disorders varies from early death in infancy (usually because of respiratory insufficiency) to prolonged survival. ${ }^{2}$ Congenital heart disease (CHD) is not generally recognised to be a feature of these conditions although there are isolated reports of cardiac malpositions. ${ }^{34}$

We report two familial cases of spondylothoracic dysostosis where complex congenital heart disease also occurred.

Department of Fetal Cardiology, 15th Floor, Guy's Hospital, St Thomas Street, London SE1 9RT, UK

J M Simpson

G K Sharland

Paediatric

Cardiopathology Unit,

Guy's Hospital,

London SE1 9RT, UK

A Cook

N L K Fagg

Department of

Obstetrics and

Gynaecology,

The General Hospital, St Helier,

Jersey JE2 3QS

N A MacLachlan

Correspondence to: Dr Simpson.

Received 11 November 1994 Revised version accepted for publication 20 April 1995

\section{Case reports}

MATERNAL HISTORY

The affected fetuses were the third and fourth pregnancies of a 35 year old white woman. The parents were non-consanguineous, the mother was not diabetic, and there was no family history of congenital heart disease. The first pregnancy had resulted in a first trimester spontaneous abortion and the second pregnancy had produced a normal male infant.

\section{CASE 1}

A routine maternal ultrasound scan at 23 weeks' gestation showed an abnormal four chamber view of the fetal heart. Detailed fetal echocardiography showed a complete atrioventricular septal defect with marked left ventricular dominance. The aorta overrode the interventricular septum and the pulmonary artery was atretic. In view of the cardiac findings the pregnancy was terminated at 24 weeks' gestation. A fetal blood sample was obtained and showed a normal male karyotype.

The male fetus was severely dysmorphic with a short, wide neck, a short trunk, and very low set ears. The right lung was composed of two lobes partially subdivided. The left lung was composed of a single lobe.

Necropsy confirmed the above cardiac findings: in addition there was a retro-oesophageal right subclavian artery. The lungs were supplied by aortic collateral vessels. There was normal atrial arrangement.

Multiple skeletal abnormalities were noted including fused vertebrae, multiple hemivertebrae, and two butterfly vertebrae. There were nine ribs on each side which were fused posteriorly. There was absent ossification of the middle phalanges of the feet. The postmortem $x$ ray of the thorax and spine is shown in fig 1 .

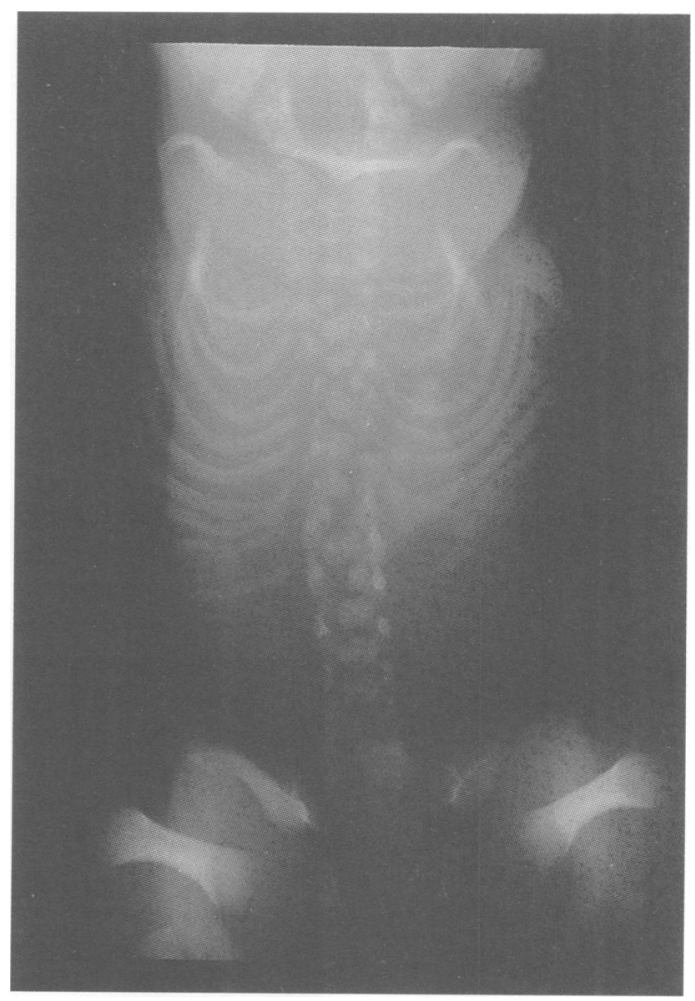

Figure 1 Skeletal malformations in a 23 week fetus (case 1). There are nine ribs on each side and multiple vertebral abnormalities including fusions and hemivertebrae (see text). 


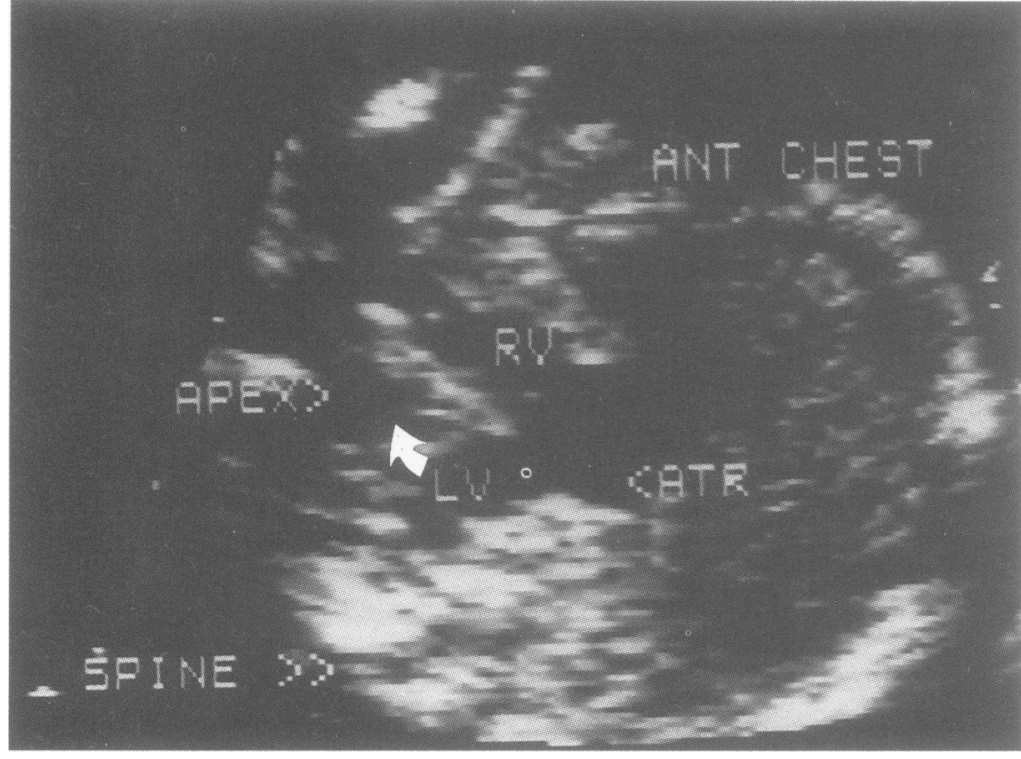

Figure 2 Four chamber echocardiographic view of the fetal heart. The position of the heart is markedly abnormal with the heart lying entirely in the left chest and the apex pointing posterolaterally. The right ventricle (RV) is larger than the left (LV). There is a complete atrioventricular septal defect. No septation of the atria (ATR) can be seen.

\section{CASE 2}

Ultrasound scanning during the second affected pregnancy indicated abnormal vertebrae, a small thorax, and an abnormal four chamber view of the fetal heart. Detailed fetal echocardiography was performed at 19 weeks' gestation and confirmed an abnormal orientation of the heart with the apex directed posterolaterally into the left axilla. There was a complete atrioventricular septal defect with right ventricular dominance (fig 2). The pulmonary artery was atretic and the aorta arose anteriorly from the

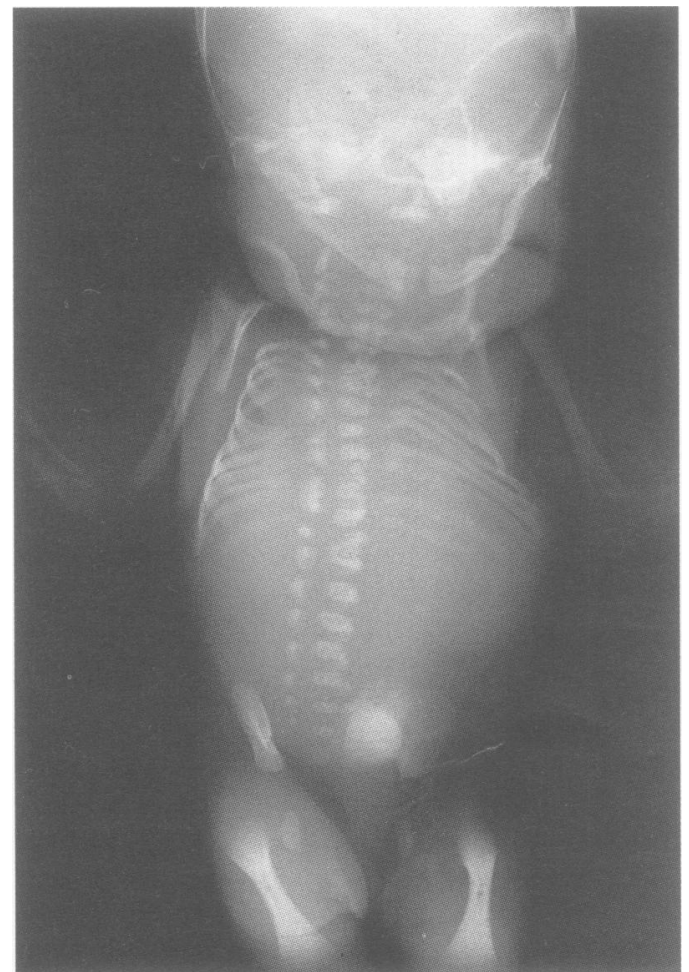

Figure 3 Radiological abnormalities in a 19 week fetus (case 2). There are 11 ribs on each side with posterior fusion of some. Severe vertebral abnormalities and a short sacrum can also be seen. morphological right ventricle. In view of these cardiac defects, the pregnancy was terminated. Skin and muscle biopsies showed a normal female karyotype in $91 \%$ of cells, but six out of 65 cells $(9 \%)$ had an additional small marker chromosome. Attempts to elucidate the nature of the marker chromosome were unsuccessful as subsequent harvests of metaphases did not show the marker. In situ hybridisation studies using the cosmid EO showed no evidence of a deletion in chromosome 22. The parents declined blood sampling for karyotyping.

Necropsy found a mildly hydropic female fetus with a short, wide neck, a short trunk, and mild micrognathia. The lengths of the fingers were abnormal with the ring finger being the longest followed by the fifth, middle, and index finger. There was a single palmar crease on the left hand.

The right lung had three lobes but there was complete agenesis of the left lung and left bronchus. This probably accounted for the abnormal position of the heart in the left side of the chest. There was the usual atrial arrangement. The echocardiographically diagnosed cardiac abnormalities were confirmed at necropsy.

Radiological examination showed severe abnormalities of the vertebrae and ribs together with a short thorax. There were multiple fused vertebrae, hemivertebrae, and "butterfly" vertebrae. The ribs were fused posteriorly and there were 11 ribs on each side. The sacrum was short. In the feet there was absent ossification of the middle row of phalanges but normal ossification of the distal phalanges. The middle phalanges of the fingers were hypoplastic. The skull and vault were normally ossified. Postmortem $x$ ray is shown in fig 3 .

\section{Discussion}

Our two cases showed the short neck, short trunk, and severe skeletal malformations which are characteristic of spondylothoracic dysostosis. The karyotype in the first affected fetus was normal and it would seem unlikely that the occurrence of a small marker chromosome in a minority of cells in the second fetus would explain the abnormalities seen. The occurrence of two cases from non-consanguineous and unaffected parents suggests an autosomal recessive mode of inheritance.

Cardiac malformations are not generally recognised to be a feature of spondylothoracic dysostosis. ${ }^{3}$ Mesocardia and dextrocardia have been described in children with spondylocostal dysostosis, ${ }^{34}$ but in those cases there was no intracardiac abnormality. The occurrence of congenital heart disease in both fetuses reported here suggests that cardiac malformations may occasionally be a feature of spondylothoracic dysostosis. Both the fetuses in this report were terminated in view of the cardiac malformations. Vertebral abnormalities were seen on antenatal ultrasound scans in the second affected fetus where they were specifically sought. This is consistent with the observations of Tolmie et $a l^{5}$ who described the ultrasound features of the condition in the second trimester. 
Prognostication is extremely difficult in spondylothoracic dysostosis and prolonged survival has been described even in children with severe skeletal abnormalities. ${ }^{2}$ Detailed cardiac ultrasound may provide useful prognostic information in both prenatally and postnatally diagnosed cases of spondylothoracic dysostosis.

We are most grateful to Dr Christine Hall, Institute of Child Health, London, for her radiological opinion of the postmortem $x$ rays of both cases.
1 Karnes PS, Day D, Berry SA, Pierpont MEM. Jarcho-Levin syndrome: four new cases and classification of subtypes. Am $\mathcal{Y}$ Med Genet 1991

2 McCall CP, Hudgins L, Cloutier M, Greenstein RM, Cassidy SB. Jarcho-Levin syndrome: unusual survival in a classic case. Am J Med Genet 1994;49:328-32.

3 Ohzeki T, Shiraishi M, Matsumoto Y, et al. Sporadic occurrence of spondylocostal dysplasia and mesocardia in Japanese girl. Am $\mathcal{\exists}$ Med Genet 1990;37:427-8.

4 Sharma AK, Phadke SR. Another case of spondylocostal dysplasia and severe anomalies: a diagnostic and counselling dilemma. Am f Med Genet 1994;50:383-4.

5 Tolmie JL, Whittle MJ, McNay MB, Gibson AAM, Connor JM. Second trimester prenatal diagnosis of the JarchoLevin syndrome. Prenat Diagn 1987;7:129-34. 